\title{
Cognitive Cooperative Networks in Dual-Hop Asymmetric Fading Channels
}

\author{
Trung Q. Duong*, Tran Trung Duy ${ }^{\dagger}$, Michail Matthaiou ${ }^{\ddagger}$, Theodoros Tsiftsis ${ }^{\S}$, and George K. Karagiannidis ${ }^{\llbracket}$ \\ *Blekinge Institute of Technology, Karlskrona, Sweden (e-mail: quang.trung.duong@bth.se) \\ ${ }^{T}$ University of Ulsan, Ulsan, Korea (e-mail: trantrungduy@mail.ulsan.ac.kr) \\ ${ }^{\ddagger}$ Chalmers University of Technology, Gothenburg, Sweden (e-mail: michail.matthaiou@chalmers.se)

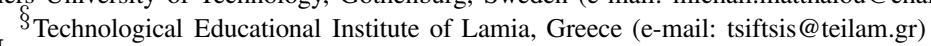 \\ 9 Aristotle University of Thessaloniki, Thessaloniki, Greece (e-mail: geokarag@auth.gr)
}

\begin{abstract}
Previous works on cognitive relay networks (CRNs) considered only symmetric fading channels. However, in practical wireless propagation scenarios, it is likely that the channels of the secondary user (SU) and primary user (PU) may undergo different fading characteristics. In this paper, we assume that the channels of the secondary network (SU-source $\rightarrow$ SU-relay $\rightarrow$ SUdestination) are subject to Rician fading, whereas the channels of the link from the SU to the PU experience Rayleigh fading. Based on this framework, the end-to-end outage probability (OP) of CRNs is investigated for two different relaying schemes: i) in the absence of the direct link with decode-and-forward (DF) protocol and ii) in the presence of the direct link with incremental DF protocol. In particular, we derive both exact and asymptotic OP expressions for the considered CRNs. Our analysis reveals important insights into the impact of fading parameters on the CRN performance under distinct fading distributions.
\end{abstract}

\section{INTRODUCTION}

By allowing the secondary user (SU) to concurrently occupy the radio frequency spectrum, which is licensed to the primary user (PU), cognitive radio networks with spectrum-sharing is an emerging technology to overcome the inefficient utilization of scarce radio frequency spectrum [1]-[7]. Recently, the use of amplify-and-forward (AF) and decode-and-forward (DF) relaying protocols has been incorporated into the cognitive radio paradigm to improve the secondary system performance. Several works have investigated the performance of cognitive cooperative networks (CCNs) over various fading channels [8]-[10]. Specifically, in [8] the outage performance of CCNs with AF relaying has been studied for Rayleigh fading channels. More recently, the works in [9] and [10] have analyzed the CCN performance in Nakagami- $m$ fading channels with DF and AF relaying, respectively. Although these previously published works offered important understandings of CCNs, all of them assumed symmetric fading conditions for the secondary and primary links.

In addition to the non line-of-sight (non-LoS) fading model, i.e., Rayleigh and Nakagami- $m$, the Rician distribution is widely employed to characterize the wireless channels with line-of-sight (LoS) or specular component [11]. On top of this, in practical wireless propagation scenarios, the links of relaying networks may experience different fading characteristics [12]. Motivated by this fact, the performance of non-cognitive relay networks under asymmetric fading channels has been considered in [13], [14]. In particular, the performance of asymmetric fading channels with Rayleigh and Rician fading has been analyzed for semi-blind and channel state information (CSI)-assisted AF relaying in [13] and [14], respectively.

For CCNs, the links of the SU and PU are highly likely to experience different fading characteristics. As such, in this paper, we assume that the secondary relay terminal can be strategically located by the operator [12], which makes the links from the secondary source to secondary relay and to secondary destination experience LoS propagation. As a result, the channels for these links SU-source $\rightarrow$ SU-relay and SU-relay $\rightarrow$ SU-destination can be modeled via the Rician distribution, while the links between SU and PU experience Rayleigh fading. We point out that the channel for the link SU-source $\rightarrow$ SU-destination is also modeled via the Rayleigh distribution. This assumption is sufficiently realistic in urban environments, where the source and destination are normally in non-LoS, such that multiple wavefronts impinge on the latter. For the sake of generality and to pursue a comparative study, we consider two different CCN configurations, with and without direct communication pertaining to the link from the secondary source to the secondary destination. Specifically, for the case without SU's direct link, namely PR1, conventional relaying with DF operation is assumed, whereas for the case with direct link, namely PR2, the incremental DF relaying is utilized to enhance the secondary system performance. In the following, we derive new, analytical expressions for the exact and asymptotic outage probability (OP) of both considered schemes. One of the paper's significant contributions is the asymptotic analysis which reveals the diversity and coding gains of the two considered systems.

The rest of the paper is organized as follows. In Section II, we first present the system and channel model for the two considered protocols in a spectrum-sharing environment. The OP performance is obtained in Section III. Numerical and simulation results are provided in Section IV. Conclusions are drawn in Section V.

\section{System AND Channel Models}

The considered cognitive network consists of a secondary source (S), a secondary destination (D), a secondary relay (R) and a primary receiver $(\mathrm{PU})$, as shown in Fig. 1. Let $d_{0}, d_{1}, d_{2}, d_{3}$, and $d_{4}$ be the distances $\mathrm{S} \rightarrow \mathrm{D}, \mathrm{S} \rightarrow \mathrm{R}$, $\mathrm{R} \rightarrow \mathrm{D}, \mathrm{S} \rightarrow \mathrm{PU}$, and $\mathrm{R} \rightarrow \mathrm{PU}$, respectively. We also denote 


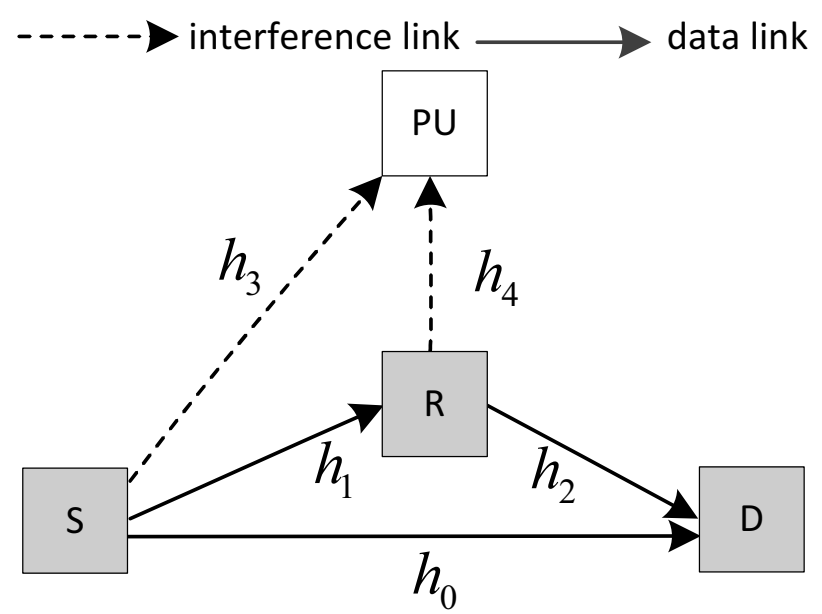

Fig. 1. System model for a cognitive DF relaying network.

$h_{0}, h_{1}, h_{2}, h_{3}$, and $h_{4}$ the corresponding channel coefficients for the links $\mathrm{S} \rightarrow \mathrm{D}, \mathrm{S} \rightarrow \mathrm{R}, \mathrm{R} \rightarrow \mathrm{D}, \mathrm{S} \rightarrow \mathrm{PU}$, and $\mathrm{R} \rightarrow \mathrm{PU}$, respectively. Here, it is assumed that the channels $h_{1}$ and $h_{2}$ follow the Rician distribution while $h_{0}, h_{3}$, and $h_{4}$ are subject to Rayleigh fading. Hence, $\gamma_{j}=\left|h_{j}\right|^{2}, j \in\{0,3,4\}$ is an exponential random variable (RV) with parameter $\lambda_{j}$. On the other hand, $\gamma_{i}=\left|h_{i}\right|^{2}, i \in\{1,2\}$, is a non-central chi-square distributed RV, whose cumulative distribution function (CDF) and probability density function (PDF) are respectively given by

$$
\begin{aligned}
& F_{\gamma_{i}}(x)=1-Q_{1}\left(\sqrt{2 K_{i}}, \sqrt{2 \psi_{i} x}\right) \\
& f_{\gamma_{i}}(x)=\left(1+K_{i}\right) \lambda_{i} e^{-K_{i}-\psi_{i} x} I_{0}\left(2 \sqrt{K_{i} \psi_{i} x}\right)
\end{aligned}
$$

where $\psi_{i} \triangleq\left(1+K_{i}\right) \lambda_{i}, Q_{1}(\cdot)$, and $I_{0}(\cdot)$ are the Marcum$Q$ function [15, Eq. (4.10)] and modified Bessel function of the first kind [16, Eq. (8.445)], respectively. In addition, $K_{i}$ is the Rician $K$-factor and $\lambda_{i}=1 / \bar{\gamma}_{i}$, where $\bar{\gamma}_{i}$ is the expected value of $\gamma_{i}$. To take path-loss into account, we model $\lambda_{k}, k \in$ $\{1,2,3,4,5\}$ as $\lambda_{k}=d_{k}^{\beta}$, where $\beta$ is the path-loss exponent.

\section{A. CCN without Direct Link}

In a cognitive network, $\mathrm{S}$ and $\mathrm{R}$ must adapt their transmit power, $P_{\mathrm{S}}$ and $P_{\mathrm{R}}$, respectively, so that the interference caused at PU is lower than a predefined interference level $I_{t h}$. Specifically, their transmit powers must be lower than a maximum threshold $P_{t h}[10]$

$$
P_{\mathrm{S}}^{\max }=\min \left(P_{t h}, \frac{I_{t h}}{\gamma_{3}}\right), \quad P_{R}^{\max }=\min \left(P_{t h}, \frac{I_{t h}}{\gamma_{4}}\right) .
$$

For the PR1 protocol, the data transmission is divided into two time slots. In the first time slot, $\mathrm{S}$ broadcasts its data $x$ to $\mathrm{R}$ as

$$
y_{\mathrm{SR}}=\sqrt{P_{\mathrm{S}}^{\max }} h_{1} x+n_{\mathrm{SR}},
$$

where $n_{\mathrm{SR}}$ is the zero-mean Gaussian noise at R. From (4), the instantaneous signal-to-noise ratio (SNR) received at $\mathrm{R}$ is given as

$$
\Psi_{\mathrm{SR}}=P_{\mathrm{S}}^{\max }\left|h_{1}\right|^{2} / N_{0}=\min \left(\gamma_{P}, \frac{\gamma_{I}}{\gamma_{3}}\right) \gamma_{1},
$$

where $N_{0}$ is the variance of the Gaussian noise, which is assumed to be the same at all receivers, i.e., $\mathrm{R}$ and $\mathrm{D}$, while $\gamma_{P}=P_{t h} / N_{0}$, and $\gamma_{I}=I_{t h} / N_{0}$. Without loss of generality, we assume that $\gamma_{I}=\mu \gamma_{P}$, where $\mu$ is a positive constant.

If $\mathrm{R}$ can successfully decode the signal, it will forward the decoded signal to $\mathrm{D}$ at the second time slot. As such, the signal received at $D$, due to the transmission of $R$, is expressed as

$$
y_{\mathrm{RD}}=\sqrt{P_{\mathrm{R}}^{\max }} h_{2} x+n_{\mathrm{RD}},
$$

where $n_{\mathrm{RD}}$ is the Gaussian noise at D. Therefore, the SNR for the second-hop is given by

$$
\Psi_{\mathrm{RD}}=P_{\mathrm{R}}^{\max }\left|h_{2}\right|^{2} / N_{0}=\min \left(\gamma_{P}, \frac{\gamma_{I}}{\gamma_{4}}\right) \gamma_{2} .
$$

The end-to-end SNR at D is therefore written as [9]

$$
\Psi_{\mathrm{e} 2 \mathrm{e}}^{\mathrm{PR} 1}=\min \left(\Psi_{\mathrm{SR}}, \Psi_{\mathrm{RD}}\right) .
$$

\section{B. CCN with Direct Link: Incremental Relaying}

For the cognitive relaying protocol with incremental relaying, named PR2, if D can successfully decode the S's signal, it feeds back an ACK to S and R to inform the decoding status. In this case, if the transmission is successful, $\mathrm{R}$ does nothing. Otherwise, D sends a NACK message to request a retransmission from $R$. If $R$ can successfully decode the signal, it will forward the decoded signal to $\mathrm{D}$ at the second time slot. In the case that $\mathrm{R}$ cannot receive the signal correctly, it will keep silent and the signal will be dropped. Therefore, the end-to-end SNR at D can be written as [17]

$$
\Psi_{\mathrm{e} 2 \mathrm{e}}^{\mathrm{PR} 2}=\left\{\begin{array}{cc}
\Psi_{\mathrm{SD}} & \text { if } \Psi_{\mathrm{SD}} \geq \varphi_{t h} \\
\min \left(\Psi_{\mathrm{SR}}, \Psi_{\mathrm{RD}}\right) & \text { if } \Psi_{\mathrm{SD}}<\varphi_{t h},
\end{array}\right.
$$

where $\varphi_{t h}$ is a predefined threshold at the relay and $\Psi_{\mathrm{SD}}$ is the instantaneous SNR for the direct link $\mathrm{S} \rightarrow \mathrm{D}$, given as

$$
\Psi_{\mathrm{SD}}=\min \left(\gamma_{P}, \frac{\gamma_{I}}{\gamma_{3}}\right) \gamma_{0}
$$

\section{Outage Probability: Exact And Asymptotic ANALYSIS}

In this paper, we assume that the data transmission is in outage if the instantaneous end-to-end SNRs are lower than a predefined threshold level $\varphi_{t h}$.

\section{A. CCN without Direct Link}

The OP of the PR1 protocol can be expressed as follows: $\mathrm{P}_{\text {out }}^{\mathrm{PR} 1}=1-\left[1-\operatorname{Pr}\left(\Psi_{\mathrm{SR}}<\varphi_{t h}\right)\right]\left[1-\operatorname{Pr}\left(\Psi_{\mathrm{RD}}<\varphi_{t h}\right)\right]$. 
Considering the probability $\operatorname{Pr}\left[\Psi_{\mathrm{RD}}<\varphi_{t h}\right]$ in (11), it can be formulated as

$$
\begin{aligned}
& \operatorname{Pr}\left(\Psi_{\mathrm{RD}}<\varphi_{t h}\right)=\operatorname{Pr}\left(\min \left(1, \mu / \gamma_{4}\right) \gamma_{P} \gamma_{2}<\varphi_{t h}\right) \\
& =\underbrace{\operatorname{Pr}\left(\gamma_{4}<\mu, \gamma_{2}<\frac{\varphi_{t h}}{\gamma_{P}}\right)}_{P_{1}^{\text {out }}}+\underbrace{\operatorname{Pr}\left(\gamma_{4} \geq \mu, \gamma_{2}<\frac{\varphi_{t h}}{\mu \gamma_{P}} \gamma_{4}\right)}_{P_{2}^{\text {out }}} .
\end{aligned}
$$

Due to the independence of $\gamma_{2}$ and $\gamma_{4}$, by using the CDF of $\gamma_{2}$ in (1), we can calculate the probability $P_{1}^{\text {out }}$ in (12) as

$$
P_{1}^{\text {out }}=\left(1-e^{-\lambda_{4} \mu}\right)\left[1-Q_{1}\left(\sqrt{2 K_{2}}, \sqrt{\frac{2 \psi_{2} \varphi_{t h}}{\gamma_{P}}}\right)\right] .
$$

Now, considering $P_{2}^{\text {out }}$ in (12), we have

$$
\begin{aligned}
& P_{2}^{\text {out }}=\int_{\mu}^{\infty} f_{\gamma_{4}}(x) F_{\gamma_{2}}\left(\frac{\varphi_{t h}}{\mu \gamma_{P}} x\right) d x=e^{-\lambda_{4} \mu} \\
& -\int_{\mu}^{\infty} \lambda_{4} e^{-\lambda_{4} x} Q_{1}\left(\sqrt{2 K_{2}}, \sqrt{\frac{2 \psi_{2} \varphi_{t h}}{\mu \gamma_{P}} x}\right) d x .
\end{aligned}
$$

Next, the Marcum- $Q$ function of the first order can be expressed by an infinite series with the help of [15, Eq. (4.35)] and [16, Eq. (8.445)]; applying this expression in (14), we obtain

$$
\begin{aligned}
P_{2}^{\text {out }} & =e^{-\lambda_{4} \mu}-\lambda_{4} \mu e^{-K_{2}-\lambda_{4} \mu} \sum_{m=0}^{\infty} \sum_{n=0}^{\infty} \sum_{t=0}^{n}\left[\frac{\psi_{2}^{n}}{(m+n) ! t !}\right. \\
& \left.\times \frac{\varphi_{t h}^{n}}{\gamma_{P}^{t-1}} \frac{K_{2}^{m+n} e^{-\frac{\psi_{2} \varphi_{t h}}{\gamma_{P}}}}{\left(\lambda_{4} \mu \gamma_{P}+\psi_{2} \varphi_{t h}\right)^{n+1-t}}\right] .
\end{aligned}
$$

From (12), (13) and (15), we obtain an exact expression for $\operatorname{Pr}\left(\Psi_{\mathrm{RD}}<\varphi_{t h}\right)$ which is shown in (16) at the top of next page. ${ }^{1}$ Note that by replacing $\lambda_{2}, \lambda_{4}$ and $K_{2}$ in (16) with $\lambda_{1}$, $\lambda_{3}$ and $K_{1}$, respectively, we can obtain an exact expression for $\operatorname{Pr}\left(\Psi_{\mathrm{SR}}<\varphi_{t h}\right)$; then, substituting the obtained results into (11), we have an exact expression for $\mathrm{P}_{\text {out }}^{\mathrm{PR} 1}$.

Proposition 1: Without maximum transmit power constraint $\gamma_{P}$, the outage probability of the PR1 protocol can be expressed by an exact closed-form expression as follows:

$$
\begin{aligned}
\mathrm{P}_{\text {out }}^{\mathrm{PR} 1} \stackrel{\gamma_{P} \rightarrow \infty}{=} 1 & -\left[1-\frac{\psi_{1} \varphi_{t h} e^{-\frac{K_{1} \lambda_{3} \gamma_{I}}{\psi_{1} \varphi_{t h}+\lambda_{3} \gamma_{I}}}}{\psi_{1} \varphi_{t h}+\lambda_{3} \gamma_{I}}\right] \\
\times & {\left[1-\frac{\psi_{2} \varphi_{t h} e^{-\frac{K_{2} \lambda_{4} \gamma_{I}}{\psi_{2} \varphi_{t h}+\lambda_{4} \gamma_{I}}}}{\psi_{2} \varphi_{t h}+\lambda_{4} \gamma_{I}}\right] . }
\end{aligned}
$$

Proof: Without maximum transmit power constraint, $P_{t h}$, the OP $\operatorname{Pr}\left(\Psi_{\mathrm{RD}}<\varphi_{t h}\right)$ in this case is obtained by taking the limit of (16) as $\gamma_{P}$ goes to infinity. Indeed, after taking the

\footnotetext{
${ }^{1}$ Although the final expression is given in infinite series, our numerical results show that its convergence is very fast, requiring only few terms to approach the exact results.
}

limit and some manipulations, we can rewrite (16) as follows:

$$
\begin{aligned}
& \lim _{\gamma_{P} \rightarrow \infty} \operatorname{Pr}\left(\Psi_{\mathrm{RD}}<\varphi_{t h}\right)=1-\frac{\lambda_{4} \gamma_{I} e^{-K_{2}}}{\psi_{2} \varphi_{t h}+\lambda_{4} \gamma_{I}} \\
& \times \sum_{m=0}^{\infty} \sum_{n=0}^{\infty} \frac{K_{2}^{m+n}}{(m+n) !} \frac{\left(\psi_{2} \varphi_{t h}\right)^{n}}{\left(\psi_{2} \varphi_{t h}+\lambda_{4} \gamma_{I}\right)^{n}} .
\end{aligned}
$$

Considering the infinite series sum in (18), we get

$\sum_{m=0}^{\infty} \sum_{n=0}^{\infty} \frac{K_{2}^{m+n}}{(m+n) !} \frac{\left(\psi_{2} \varphi_{t h}\right)^{n}}{\left(\psi_{2} \varphi_{t h}+\lambda_{4} \gamma_{I}\right)^{n}}=\sum_{n=0}^{\infty} \frac{\left(\psi_{2} \varphi_{t h}\right)^{n}}{\left(\psi_{2} \varphi_{t h}+\lambda_{4} \gamma_{I}\right)^{n}}$

$\times \sum_{m=0}^{\infty} \frac{K_{2}^{m}}{m !}-\sum_{n=1}^{\infty} \frac{\left(\psi_{2} \varphi_{t h}\right)^{n}}{\left(\psi_{2} \varphi_{t h}+\lambda_{4} \gamma_{I}\right)^{n}} \sum_{m=0}^{n-1} \frac{K_{2}^{m}}{m !}$.

In addition, we have

$$
\begin{aligned}
& \sum_{n=0}^{\infty} \frac{\left(\psi_{2} \varphi_{t h}\right)^{n}}{\left(\psi_{2} \varphi_{t h}+\lambda_{4} \gamma_{I}\right)^{n}} \sum_{m=0}^{\infty} \frac{K_{2}^{m}}{m !}=\frac{\left(\psi_{2} \varphi_{t h}+\lambda_{4} \gamma_{I}\right) e^{K_{2}}}{\lambda_{4} \gamma_{I}} \\
& \sum_{n=1}^{\infty} \frac{\left(\psi_{2} \varphi_{t h}\right)^{n}}{\left(\psi_{2} \varphi_{t h}+\lambda_{4} \gamma_{I}\right)^{n}} \sum_{m=0}^{n-1} \frac{K_{2}^{m}}{m !}=\frac{\psi_{2} \varphi_{t h}}{\lambda_{4} \gamma_{I}} e^{\frac{K_{2} \psi_{2} \varphi_{t h}}{\psi_{2} \varphi_{t h}+\lambda_{4} \gamma_{I}}}
\end{aligned}
$$

Substituting (19), (20), and (21) into (18), we obtain

$$
\lim _{\gamma_{P} \rightarrow \infty} \operatorname{Pr}\left(\Psi_{\mathrm{RD}}<\varphi_{t h}\right)=\frac{\psi_{2} \varphi_{t h} e^{-\frac{K_{2} \lambda_{4} \gamma_{I}}{\psi_{2} \varphi_{t h}+\lambda_{4} \gamma_{I}}}}{\psi_{2} \varphi_{t h}+\lambda_{4} \gamma_{I}} .
$$

Now, let us replace $\lambda_{2}, \lambda_{4}$ and $K_{2}$ in (22) by $\lambda_{1}, \lambda_{3}$ and $K_{1}$, respectively, to obtain an expression for $\operatorname{Pr}\left(\Psi_{\mathrm{SR}}<\varphi_{t h}\right)$. Finally, substituting the obtained results for $\operatorname{Pr}\left(\Psi_{\mathrm{SR}}<\varphi_{t h}\right)$ and $\operatorname{Pr}\left(\Psi_{\mathrm{RD}}<\varphi_{\text {th }}\right)$ into (11), we get (17).

Proposition 2: At very large $\gamma_{P}$ and $\gamma_{I}$ values, i.e., $\gamma_{P}, \gamma_{I} \rightarrow \infty$, we can express $\mathrm{P}_{\text {out }}^{\mathrm{PR} 1}$ as

$$
\begin{aligned}
& \mathrm{P}_{\text {out }}^{\mathrm{PR} 1} \stackrel{\substack{\gamma_{P} \rightarrow \infty \\
\gamma_{I} \rightarrow \infty}}{=}\left[\psi_{1} e^{-K_{1}}\left(1+\frac{e^{-\lambda_{3} \mu}}{\lambda_{3} \mu}\right)\right. \\
& \left.+\psi_{2} e^{-K_{2}}\left(1+\frac{e^{-\lambda_{4} \mu}}{\lambda_{4} \mu}\right)\right] \frac{\varphi_{t h}}{\gamma_{P}}+O\left(\frac{1}{\gamma_{P}^{2}}\right) .
\end{aligned}
$$

Proof: From (16), by using a Taylor expansion, we can obtain an approximating expression for $\operatorname{Pr}\left(\Psi_{\mathrm{RD}}<\varphi_{t h}\right)$ at very large $\gamma_{P}$ and $\gamma_{I}$ value (or $1 / \gamma_{P} \rightarrow 0,1 / \gamma_{I} \rightarrow 0$ ) as

$$
\operatorname{Pr}\left(\Psi_{\mathrm{RD}}<\varphi_{t h}\right)=s_{0}+\frac{s_{1}}{\gamma_{P}}+O\left(\frac{1}{\gamma_{P}^{2}}\right) .
$$

In (24), $s_{0}$ is calculated by

$$
s_{0}=\lim _{1 / \gamma_{P} \rightarrow 0} \operatorname{Pr}\left(\Psi_{\mathrm{RD}}<\varphi_{t h}\right)=0 .
$$

In addition, $s_{1}$ in (24) can be formulated as

$$
\begin{aligned}
& s_{1}=\lim _{\frac{1}{\gamma_{P}} \rightarrow 0} \frac{\partial \operatorname{Pr}\left(\Psi_{\mathrm{RD}}<\varphi_{t h}\right)}{\partial\left(\frac{1}{\gamma_{P}}\right)} \\
& =\left(1-e^{-\lambda_{4} \mu}\right) \psi_{2} e^{-K_{2}} \varphi_{t h}+\lambda_{4} \mu e^{-K_{2}-\lambda_{4} \mu}\left[\sum_{m=0}^{\infty} \frac{K_{2}^{m} \psi_{2}}{m !}\right. \\
& \left.\times \frac{\left(1+\lambda_{4} \mu\right)}{\lambda_{4}^{2} \mu^{2}}-\sum_{m=0}^{\infty} \frac{K_{2}^{m+1} \psi_{2}}{(m+1) !}\left(\frac{1}{\lambda_{4}^{2} \mu^{2}}+\frac{1}{\lambda_{4} \mu}\right)\right] \varphi_{t h} .
\end{aligned}
$$




$$
\begin{aligned}
\operatorname{Pr}\left(\Psi_{\mathrm{RD}}<\varphi_{t h}\right) & =\left(1-e^{-\lambda_{4} \mu}\right)\left[1-Q_{1}\left(\sqrt{2 K_{2}}, \sqrt{\frac{2 \psi_{2} \varphi_{t h}}{\gamma_{P}}}\right)\right]+e^{-\lambda_{4} \mu} \\
& -\lambda_{4} \mu e^{-K_{2}-\lambda_{4} \mu} \sum_{m=0}^{\infty} \sum_{n=0}^{\infty} \sum_{t=0}^{n} \frac{K_{2}^{m+n} \psi_{2}^{n}}{(m+n) ! t !} \frac{\varphi_{t h}^{n}}{\gamma_{P}^{t-1}} \frac{e^{-\psi_{2} \varphi_{t h} / \gamma_{P}}}{\left(\lambda_{4} \mu \gamma_{P}+\psi_{2} \varphi_{t h}\right)^{n+1-t}} .
\end{aligned}
$$

Using the fact that

$$
\sum_{m=0}^{\infty} \frac{K_{2}^{m}}{m !}=e^{K_{2}}, \quad \sum_{m=0}^{\infty} \frac{K_{2}^{m+1}}{(m+1) !}=e^{K_{2}}-1,
$$

we obtain

$$
s_{1}=\psi_{2} e^{-K_{2}}\left(1+\frac{e^{-\lambda_{4} \mu}}{\lambda_{4} \mu}\right) \varphi_{t h} .
$$

Combining (24), (25) with (28), we get

$$
\begin{aligned}
& \operatorname{Pr}\left(\Psi_{\mathrm{RD}}<\varphi_{t h}\right)=\psi_{2} e^{-K_{2}}\left(1+\frac{e^{-\lambda_{4} \mu}}{\lambda_{4} \mu}\right) \frac{\varphi_{t h}}{\gamma_{P}}+O\left(\frac{1}{\gamma_{P}^{2}}\right), \\
& \operatorname{Pr}\left(\Psi_{\mathrm{SR}}<\varphi_{t h}\right)=\psi_{1} e^{-K_{1}}\left(1+\frac{e^{-\lambda_{3} \mu}}{\lambda_{3} \mu}\right) \frac{\varphi_{t h}}{\gamma_{P}}+O\left(\frac{1}{\gamma_{P}^{2}}\right) .
\end{aligned}
$$

Finally, substituting (29) into (11), we get (23), which completes the proof.

Corollary 1: Without transmit power constraint, the asymptotic OP of the PR1 protocol can be given as

$$
\mathrm{P}_{\text {out }}^{\mathrm{PR} 1} \stackrel{\gamma_{P} \rightarrow \infty}{=}\left[\frac{\psi_{1} e^{-K_{1}}}{\lambda_{3}}+\frac{\psi_{2} e^{-K_{2}}}{\lambda_{4}}\right] \frac{\varphi_{t h}}{\gamma_{I}}+O\left(\frac{1}{\gamma_{I}^{2}}\right) \text {. }
$$

\section{B. CCN with Direct Link: Incremental Relaying}

In the second cognitive relaying protocol, named PR2, we assume that the incremental relaying scheme is used to relay the source's data to D [17]. With this transmission scheme, the OP of the PR2 protocol is calculated as follows:

$$
\begin{aligned}
\mathrm{P}_{\mathrm{out}}^{\mathrm{PR} 2} & =\operatorname{Pr}\left(\Psi_{\mathrm{e} 2 \mathrm{e}}^{\mathrm{PR}}<\varphi_{t h}\right)=\operatorname{Pr}\left(\Psi_{\mathrm{SD}}<\varphi_{t h}, \Psi_{\mathrm{SR}}<\varphi_{t h}\right) \\
& +\operatorname{Pr}\left(\Psi_{\mathrm{SD}}<\varphi_{t h}, \Psi_{\mathrm{RD}}<\varphi_{t h}, \Psi_{\mathrm{SR}} \geq \varphi_{t h}\right)
\end{aligned}
$$

Considering the first term $\operatorname{Pr}\left[\Psi_{\mathrm{SD}}<\varphi_{t h}, \Psi_{\mathrm{SR}}<\varphi_{t h}\right]$ in (31), it can be formulated as

$$
\begin{aligned}
& \operatorname{Pr}\left(\Psi_{\mathrm{SD}}<\varphi_{t h}, \Psi_{\mathrm{SR}}<\varphi_{t h}\right) \\
& =\underbrace{\operatorname{Pr}\left(\gamma_{3}<\mu, \gamma_{0}<\frac{\varphi_{t h}}{\gamma_{P}}, \gamma_{1}<\frac{\varphi_{t h}}{\gamma_{P}}\right)}_{P_{3}^{\text {out }}} \\
& +\underbrace{\operatorname{Pr}\left(\gamma_{3} \geq \mu, \gamma_{0}<\frac{\varphi_{\text {th }}}{\mu \gamma_{P}} \gamma_{3}, \gamma_{1}<\frac{\varphi_{t h}}{\mu \gamma_{P}} \gamma_{3}\right)}_{P_{4}^{\text {out }}} .
\end{aligned}
$$

By using the CDF of $\gamma_{0}, \gamma_{1}$ and $\gamma_{3}, P_{3}^{o u t}$ in (32) is calculated as

$$
\begin{aligned}
P_{3}^{\text {out }} & =\left(1-e^{-\lambda_{3} \mu}\right)\left(1-e^{-\lambda_{0} \frac{\varphi_{\text {th }}}{\gamma_{P}}}\right) \\
& \times\left[1-Q_{1}\left(\sqrt{2 K_{1}}, \sqrt{2 \psi_{1} \frac{\varphi_{t h}}{\gamma_{P}}}\right)\right] .
\end{aligned}
$$

For $P_{4}^{\text {out }}$ in (32), it is easy to see that

$$
P_{4}^{\text {out }}=\int_{\mu}^{\infty} f_{\gamma_{3}}(x) F_{\gamma_{1}}\left(\frac{\varphi_{t h}}{\mu \gamma_{P}} x\right) F_{\gamma_{0}}\left(\frac{\varphi_{t h}}{\mu \gamma_{P}} x\right) d x .
$$

Substituting the CDFs of $\gamma_{1}$ in (1) into (34) together with the fact that the CDF and PDF of exponential RVs $X$, for $X \in\left\{\gamma_{0}, \gamma_{3}\right\}$, are given as $F_{X}(x)=1-e^{-\lambda x}$ and $f_{X}(x)=$ $\lambda e^{-\lambda x}$, respectively, we obtain

$$
\begin{aligned}
P_{4}^{\text {out }} & =\int_{\mu}^{\infty} \lambda_{3} e^{-\lambda_{3} x}\left(1-e^{-\frac{\lambda_{0} \varphi_{t h}}{\mu \gamma_{P}} x}\right) \\
& \times\left[1-Q_{1}\left(\sqrt{2 K_{1}}, \sqrt{2 \psi_{1} \frac{\varphi_{t h}}{\mu \gamma_{P}} x}\right)\right] d x .
\end{aligned}
$$

By using the infinite series expansion of $Q_{1}(.,$.$) [15,$ Eq. (4.35)] into (35), we can obtain an expression for $P_{4}^{\text {out }}$ as

$$
\begin{aligned}
& P_{4}^{\text {out }}=e^{-\lambda_{3} \mu}-\frac{\lambda_{3} \mu \gamma_{P} e^{-\left(\lambda_{0} \varphi_{t h} / \gamma_{P}+\lambda_{3} \mu\right)}}{\lambda_{0} \varphi_{t h}+\lambda_{3} \mu \gamma_{P}}-\lambda_{3} \mu e^{-K_{1}-\lambda_{3} \mu} \\
& \times \sum_{m=0}^{\infty} \sum_{n=0}^{\infty} \sum_{t=0}^{n} \frac{K_{1}^{m+n} \psi_{1}^{n}}{(m+n) ! t !} \frac{\varphi_{t h}^{n}}{\gamma_{P}^{t-1}}\left[\frac{e^{-\psi_{1} \varphi_{t h} / \gamma_{P}}}{\left(\psi_{1} \varphi_{t h}+\lambda_{3} \mu \gamma_{P}\right)^{n+1-t}}\right. \\
& \left.-\frac{e^{-\left(\lambda_{0}+\psi_{1}\right) \varphi_{t h} / \gamma_{P}}}{\left(\lambda_{0} \varphi_{t h}+\psi_{1} \varphi_{t h}+\lambda_{3} \mu \gamma_{P}\right)^{n+1-t}}\right]
\end{aligned}
$$

From (32), (33) and (36), we have

$\operatorname{Pr}\left(\Psi_{\mathrm{SD}}<\varphi_{t h}, \Psi_{\mathrm{SR}}<\varphi_{t h}\right)=\left(1-e^{-\lambda_{3} \mu}\right)\left(1-e^{-\lambda_{0} \frac{\varphi_{t h}}{\gamma_{P}}}\right)$ $\times e^{-\lambda_{3} \mu}\left[1-Q_{1}\left(\sqrt{2 K_{1}}, \sqrt{2 \psi_{1} \frac{\varphi_{t h}}{\gamma_{P}}}\right)\right]-e^{-\left(\lambda_{0} \varphi_{t h} / \gamma_{P}+\lambda_{3} \mu\right)}$ $\times \frac{\lambda_{3} \mu \gamma_{P}}{\lambda_{0} \varphi_{t h}+\lambda_{3} \mu \gamma_{P}}-\lambda_{3} \mu e^{-K_{1}-\lambda_{3} \mu} \sum_{m=0}^{\infty} \sum_{n=0}^{\infty} \sum_{t=0}^{n} \frac{\varphi_{t h}^{n}}{\gamma_{P}^{t-1}}$ $\times \frac{K_{1}^{m+n} \psi_{1}^{n}}{(m+n) ! t !}\left[\frac{e^{-\psi_{1} \varphi_{t h} / \gamma_{P}}}{\left(\psi_{1} \varphi_{t h}+\lambda_{3} \mu \gamma_{P}\right)^{n+1-t}}\right.$

$\left.-\frac{e^{-\left(\lambda_{0}+\psi_{1}\right) \varphi_{t h} / \gamma_{P}}}{\left(\lambda_{0} \varphi_{t h}+\psi_{1} \varphi_{t h}+\lambda_{3} \mu \gamma_{P}\right)^{n+1-t}}\right]$.

Next, the second term in (31) can be written as

$$
\begin{aligned}
& \operatorname{Pr}\left(\Psi_{\mathrm{SD}}<\varphi_{t h}, \Psi_{\mathrm{RD}}<\varphi_{t h}, \Psi_{\mathrm{SR}} \geq \varphi_{t h}\right) \\
& =\operatorname{Pr}\left(\Psi_{\mathrm{SD}}<\varphi_{t h}, \Psi_{\mathrm{SR}} \geq \varphi_{t h}\right) \operatorname{Pr}\left(\Psi_{\mathrm{RD}}<\varphi_{t h}\right)
\end{aligned}
$$

Similarly, we can calculate the joint probability $\operatorname{Pr}\left(\Psi_{\mathrm{SD}}<\varphi_{t h}, \Psi_{\mathrm{SR}} \geq \varphi_{t h}\right)$ in (38) as 


$$
\begin{aligned}
& \operatorname{Pr}\left(\Psi_{\mathrm{SD}}<\varphi_{t h}, \Psi_{\mathrm{SR}} \geq \varphi_{t h}\right) \\
& =\operatorname{Pr}\left(\gamma_{3}<\mu, \gamma_{0}<\frac{\varphi_{t h}}{\gamma_{P}}, \gamma_{1} \geq \frac{\varphi_{t h}}{\gamma_{P}}\right) \\
& +\operatorname{Pr}\left(\gamma_{3} \geq \mu, \gamma_{0}<\frac{\varphi_{t h}}{\mu \gamma_{P}} \gamma_{3}, \gamma_{1} \geq \frac{\varphi_{t h}}{\mu \gamma_{P}} \gamma_{3}\right), \\
& =\left(1-e^{-\lambda_{3} \mu}\right)\left(1-e^{-\lambda_{0} \frac{\varphi_{t h}}{\gamma_{P}}}\right) Q_{1}\left(\sqrt{2 K_{1}}, \sqrt{2 \psi_{1} \frac{\varphi_{t h}}{\gamma_{P}}}\right) \\
& +\lambda_{3} \mu e^{-K_{1}-\lambda_{3} \mu} \sum_{m=0}^{+\infty} \sum_{n=0}^{+\infty} \sum_{t=0}^{n} \frac{K_{1}^{m+n} \psi_{1}^{n}}{(m+n) ! t !} \frac{\varphi_{t h}^{n}}{\gamma_{P}^{t-1}} \\
& \quad \times\left[\frac{e^{-\frac{\psi_{1} \varphi_{t h}}{\gamma_{P}}}}{\left(\psi_{1} \varphi_{t h}+\lambda_{3} \mu \gamma_{P}\right)^{n+1-t}}\right. \\
& \left.\quad-\frac{e^{-\frac{\left(\lambda_{0}+\psi_{1}\right) \varphi_{t h}}{\gamma_{P}}}}{\left(\lambda_{0} \varphi_{t h}+\psi_{1} \varphi_{t h}+\lambda_{3} \mu \gamma_{P}\right)^{n+1-t}}\right] .
\end{aligned}
$$

Finally, by plugging everything together, i.e., (16), (37), (38), and (39) into (31), the OP of the scheme PR2 over asymmetric fading channels can be obtained in closed-form expression, which is not shown here due to space-limit.

Proposition 3: Without transmit power constraint $\gamma_{P}$, the OP of the PR2 protocol can be expressed by an exact closedform expression in (40) shown at the top of next page.

Proof: Similar to the proof of Proposition 1, by taking the limit of (37) and (39) as $\gamma_{I}$ is fixed and $\gamma_{P}$ goes to infinity, we respectively obtain

$$
\begin{aligned}
& \lim _{\gamma_{P} \rightarrow \infty} \operatorname{Pr}\left(\Psi_{\mathrm{SD}}<\varphi_{t h}, \Psi_{\mathrm{SR}}<\varphi_{t h}\right)=\frac{\psi_{1} \varphi_{t h} e^{-\frac{K_{1} \lambda_{3} \gamma_{I}}{\psi_{1} \varphi_{t h}+\lambda_{3} \gamma_{I}}}}{\left(1+K_{1}\right) \lambda_{1} \varphi_{t h}+\lambda_{3} \gamma_{I}} \\
& -\frac{\lambda_{3} \gamma_{I} \psi_{1} \varphi_{t h} e^{-\frac{K_{1}\left(\lambda_{0} \varphi_{t h}+\lambda_{3} \gamma_{I}\right)}{\lambda_{0} \varphi_{t h}+\psi_{1} \varphi_{t h}+\lambda_{3} \gamma_{I}}}}{\left(\lambda_{0} \varphi_{t h}+\lambda_{3} \gamma_{I}\right)\left(\lambda_{0} \varphi_{t h}+\psi_{1} \varphi_{t h}+\lambda_{3} \gamma_{I}\right)} \\
& \quad \lim _{\gamma_{P} \rightarrow \infty} \operatorname{Pr}\left(\Psi_{\mathrm{SD}}<\varphi_{t h}, \Psi_{\mathrm{SR}} \geq \varphi_{t h}\right) \\
& =\frac{\lambda_{0} \varphi_{t h}}{\lambda_{0} \varphi_{t h}+\lambda_{3} \gamma_{I}}-\frac{\psi_{1} \varphi_{t h}}{\psi_{1} \varphi_{t h}+\lambda_{3} \gamma_{I}} e^{-\frac{K_{1} \lambda_{3} \gamma_{I}}{\psi_{1} \varphi_{t h}+\lambda_{3} \gamma_{I}}} \\
& \quad+\frac{\lambda_{3} \gamma_{I} \psi_{1} \varphi_{t h} e^{-\frac{K_{1}\left(\lambda_{0} \varphi_{t h}+\lambda_{3} \gamma_{I}\right)}{\lambda_{0} \varphi_{t h}+\psi_{1} \varphi_{t h}+\lambda_{3} \gamma_{I}}}}{\left(\lambda_{3} \gamma_{I}+\lambda_{0} \varphi_{t h}\right)\left(\lambda_{0} \varphi_{t h}+\psi_{1} \varphi_{t h}+\lambda_{3} \gamma_{I}\right)}
\end{aligned}
$$

Finally, substituting (22), (41) and (42) into (31), we obtain (40).

Proposition 4: At very large $\gamma_{P}$ and $\gamma_{I}$ values, i.e., $\gamma_{P}, \gamma_{I} \rightarrow \infty$, we can express $\mathrm{P}_{\text {out }}^{\mathrm{PR} 2}$ as in (44) at the top of next page.

Proof: First, by using the Taylor expansion for (37) for very large $\gamma_{P}$ and $\gamma_{I}$ values, we can express $\operatorname{Pr}\left(\Psi_{\mathrm{SD}}<\varphi_{t h}, \Psi_{\mathrm{SR}}<\varphi_{t h}\right)$ as follows:

$\operatorname{Pr}\left(\Psi_{\mathrm{SD}}<\varphi_{t h}, \Psi_{\mathrm{SR}}<\varphi_{t h}\right)=t_{0}+\frac{t_{1}}{\gamma_{P}}+\frac{t_{2}}{2 !} \frac{1}{\gamma_{P}^{2}}+O\left(\frac{1}{\gamma_{P}^{3}}\right)$

Similarly, $t_{0}, t_{1}$ and $t_{2}$ can be respectively given as

$$
t_{0}=\lim _{1 / \gamma_{P} \rightarrow 0} \operatorname{Pr}\left(\Psi_{\mathrm{SD}}<\varphi_{t h}, \Psi_{\mathrm{SR}}<\varphi_{t h}\right)=0 .
$$

$$
\begin{aligned}
t_{1} & =\lim _{1 / \gamma_{P} \rightarrow 0} \frac{\partial \operatorname{Pr}\left(\Psi_{\mathrm{SD}}<\varphi_{t h}, \Psi_{\mathrm{SR}}<\varphi_{t h}\right)}{\partial\left(1 / \gamma_{P}\right)}=\frac{\lambda_{0}\left(1+\lambda_{3} \mu\right)}{\lambda_{3} \mu} \\
& \times e^{-\lambda_{3} \mu}-e^{-K_{1}-\lambda_{3} \mu} \sum_{m=0}^{\infty} \frac{K_{1}^{m}}{m !} \frac{\lambda_{0}\left(1+\lambda_{3} \mu\right)}{\lambda_{3} \mu}=0 \\
t_{2} & =\lim _{1 / \gamma_{P} \rightarrow 0} \frac{\partial^{2} \operatorname{Pr}\left[\Psi_{\mathrm{SD}}<\varphi_{t h}, \Psi_{\mathrm{SR}}<\varphi_{t h}\right]}{\partial^{2}\left(1 / \gamma_{P}\right)}=2\left(1+K_{1}\right) \\
& \times \lambda_{0} \lambda_{1} e^{-K_{1}}\left(1+\frac{2+2 \lambda_{3} \mu}{\lambda_{3}^{2} \mu^{2}} e^{-\lambda_{3} \mu}\right) \varphi_{t h}^{2} .
\end{aligned}
$$

From (45) to (48), we have

$$
\begin{aligned}
& \operatorname{Pr}\left(\Psi_{\mathrm{SD}}<\varphi_{t h}, \Psi_{\mathrm{SR}}<\varphi_{t h}\right)=\left(1+K_{1}\right) \lambda_{0} \lambda_{1} e^{-K_{1}} \\
& \times\left(1+\frac{2+2 \lambda_{3} \mu}{\lambda_{3}^{2} \mu^{2}} e^{-\lambda_{3} \mu}\right) \frac{\varphi_{t h}^{2}}{\gamma_{P}^{2}}+O\left(\frac{1}{\gamma_{P}^{3}}\right) .
\end{aligned}
$$

Similarly, we also obtain

$$
\operatorname{Pr}\left[\Psi_{\mathrm{SD}}<\varphi_{t h}, \Psi_{\mathrm{SR}} \geq \varphi_{t h}\right]=\left(\lambda_{0}+\frac{\lambda_{0} e^{-\lambda_{3} \mu}}{\lambda_{3} \mu}\right) \frac{\varphi_{t h}}{\gamma_{P}} .
$$

Now, substituting (29), (49) and (50) into (31), we obtain (44).

Corollary 2: Without transmit power constraint $\gamma_{P}$, the asymptotic outage probability of the PR 2 protocol can be given as

$\mathrm{P}_{\text {out }}^{\mathrm{PR} 2} \stackrel{\gamma_{P} \rightarrow \infty}{=}\left(\frac{2 \lambda_{0} \psi_{1} e^{-K_{1}}}{\lambda_{3}^{2}}+\frac{\lambda_{0} \psi_{2} e^{-K_{2}}}{\lambda_{3} \lambda_{4}}\right) \frac{\varphi_{t h}^{2}}{\gamma_{I}^{2}}+O\left(\frac{1}{\gamma_{I}^{3}}\right)$.

Proof: By utilizing the similar approach when $\gamma_{I}$ is fixed and $\gamma_{P}$ tends to infinity, we can obtain (51), which completes the proof.

From Proposition 2 (Corollary 1) and Proposition 4 (Corollary 2), it is apparent that the diversity order of the PR1 and PR2 protocols equals 1 and 2, respectively.

\section{NumericAl Results}

In this section, we present various numerical results to verify the theoretical derivation by comparing the analysis with Monte Carlo simulations. Without loss of generality, we assume that the co-ordinates of $\mathrm{S}, \mathrm{D}, \mathrm{R}$, and $\mathrm{PU}$ are $(0,0)$, $(1,0),\left(x_{\mathrm{R}}, 0\right)$, and $\left(x_{\mathrm{PR}}, y_{\mathrm{PR}}\right)$, respectively. Hence, the corresponding distances are calculated as $d_{0}=1, d_{1}=x_{\mathrm{R}}, d_{2}=$ $1-x_{\mathrm{R}}, d_{3}=\sqrt{x_{\mathrm{PR}}^{2}+y_{\mathrm{PR}}^{2}}$, and $d_{4}=\sqrt{\left(x_{\mathrm{R}}^{2}-x_{\mathrm{PR}}\right)^{2}+y_{\mathrm{PR}}^{2}}$. In all simulations, the path-loss exponent is set as $\beta=3$ and the threshold level is fixed by $\varphi_{t h}=1$. When infinite series are being used, we truncate them at 50 terms.

In Fig. 2, we plot the OP as a function of $\gamma_{P}$ in $\mathrm{dB}$ when $\mu=1, x_{\mathrm{R}}=x_{\mathrm{PR}}=y_{\mathrm{PR}}=0.5$ and $K_{1}=K_{2}=1 \mathrm{~dB}$. It can be seen that the PR2 protocol outperforms the PR1 protocol as it utilizes the direct and relaying links to provide cooperative diversity gain.

In Fig. 3, we investigate the impact of PU's positions on the system performance when the parameters are fixed at $\mu=1$, $x_{\mathrm{R}}=0.4, K_{1}=K_{2}=1.5 \mathrm{~dB}$. As can clearly seen from this 


$$
\begin{aligned}
& \mathrm{P}_{\text {out }}^{\mathrm{PR} 2} \stackrel{\gamma_{P} \rightarrow \infty}{=} \frac{\psi_{1} \varphi_{t h}}{\psi_{1} \varphi_{t h}+\lambda_{3} \gamma_{I}} e^{-\frac{K_{1} \lambda_{3} \gamma_{I}}{\psi_{1} \varphi_{t h}+\lambda_{3} \gamma_{I}}}-\frac{\lambda_{3} \gamma_{I}}{\lambda_{0} \varphi_{t h}+\lambda_{3} \gamma_{I}} \frac{\psi_{1} \varphi_{t h} e^{-\frac{K_{1}\left(\lambda_{0} \varphi_{t h}+\lambda_{3} \gamma_{I}\right)}{\lambda_{0} \varphi_{t h}+\psi_{1} \varphi_{t h}+\lambda_{3} \gamma_{I}}}}{\lambda_{0} \varphi_{t h}+\psi_{1} \varphi_{t h}+\lambda_{3} \gamma_{I}} \\
& +\left[\frac{\lambda_{0} \varphi_{t h}}{\lambda_{0} \varphi_{t h}+\lambda_{3} \gamma_{I}}-\frac{\psi_{1} \varphi_{t h} e^{-\frac{K_{1} \lambda_{3} \gamma_{I}}{\psi_{1} \varphi_{t h}+\lambda_{3} \gamma_{I}}}}{\psi_{1} \varphi_{t h}+\lambda_{3} \gamma_{I}}+\frac{\lambda_{3} \gamma_{I}}{\lambda_{0} \varphi_{t h}+\lambda_{3} \gamma_{I}} \frac{\psi_{1} \varphi_{t h}}{\lambda_{0} \varphi_{t h}+\psi_{1} \varphi_{t h}+\lambda_{3} \gamma_{I}}\right. \\
& \left.\times e^{-\frac{K_{1}\left(\lambda_{0} \varphi_{t h}+\lambda_{3} \gamma_{I}\right)}{\lambda_{0} \varphi_{t h}+\psi_{1} \varphi_{t h}+\lambda_{3} \gamma_{I}}}\right] \frac{\psi_{2} \varphi_{t h}}{\psi_{2} \varphi_{t h}+\lambda_{4} \gamma_{I}} e^{-\frac{K_{2} \lambda_{4} \gamma_{I}}{\psi_{2} \varphi_{t h}+\lambda_{4} \gamma_{I}}}
\end{aligned}
$$

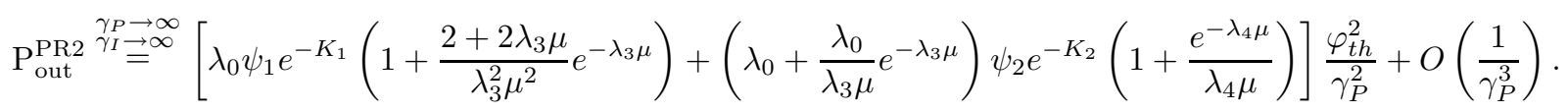

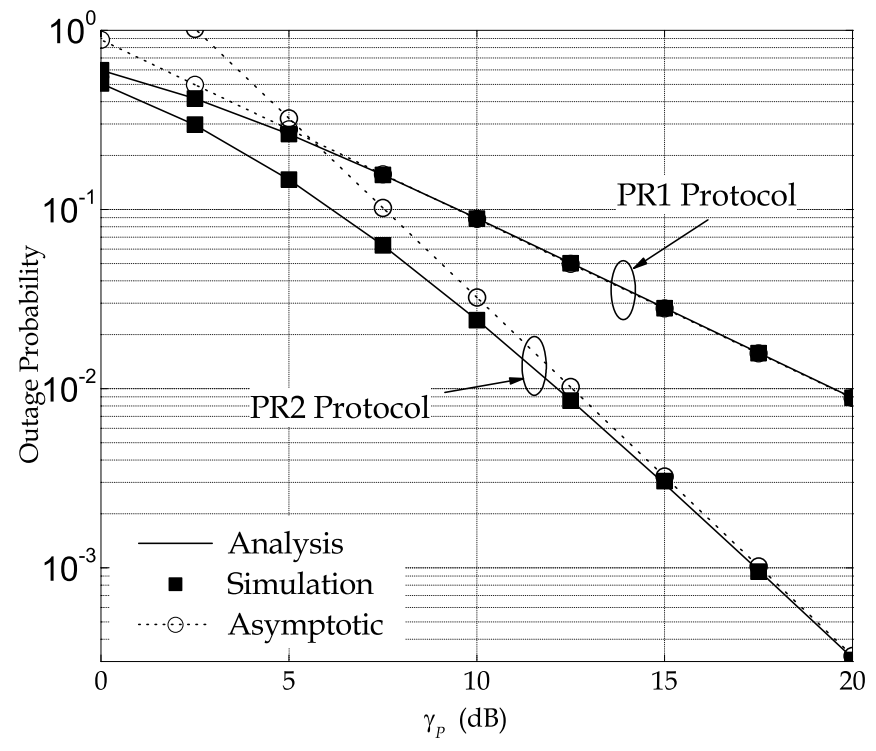

Fig. 2. The OP as a function of $\gamma_{P}$ in $\mathrm{dB}$ when $x_{\mathrm{R}}=x_{\mathrm{PR}}=y_{\mathrm{PR}}$ $=0.5$ and $K_{1}=K_{2}=1 \mathrm{~dB}$.

figure, the outage performance of PR1 and PR2 protocols is enhanced when PU moves further from the secondary source as the power constraint can be more relaxed.

In Fig. 4, we examine the impact of R's positions on the system performance when the parameters are fixed as $x_{\mathrm{PR}}=$ $y_{\mathrm{PR}}=0.5, K_{1}=K_{2}=2 \mathrm{~dB}$, and $\gamma_{P}=10 \mathrm{~dB}$. It can be seen that for each position of $\mathrm{PU}$, we can find an optimal position of the relay at which the OP yields its lowest value.

In Figs. 5- 6, we use the Golden Section Search method [18] to find the optimal position of $\mathrm{R}$ over the interval $[0,1]$ to minimize the outage performance at each fixed position of PU. The purpose of this method is to find an interval $\left[x_{R}^{L o w}, x_{R}^{U p}\right]$ which includes the optimal point $x_{R}^{*}$ and satisfies an uncertainty condition, i.e., $x_{R}^{U p}-x_{R}^{L o w} \leq \varepsilon$. In these simulations, we set $\varepsilon$ as $\varepsilon=10^{-4}$ and the optimal point $x_{R}^{*}$ is obtained as $x_{R}^{*}=\left(x_{R}^{U p}+x_{R}^{L o w}\right) / 2$. In Figs. 5, we fix $y_{\mathrm{PR}}=0.5$ while changing $x_{\mathrm{PR}}$ from 0 to 1 . We can see that

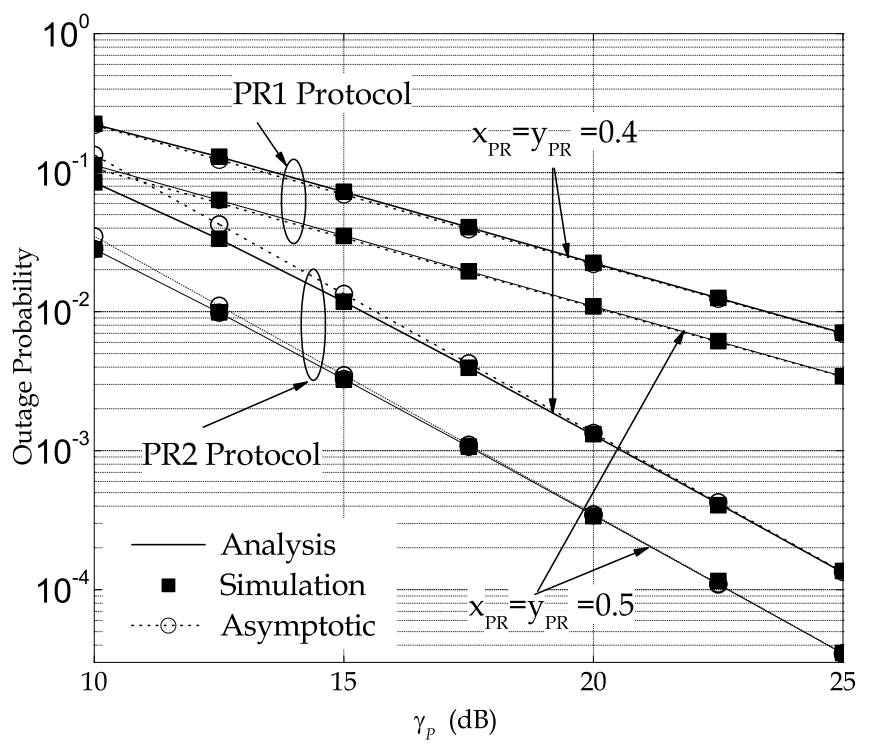

Fig. 3. The OP as a function of $\gamma_{P}$ in $\mathrm{dB}$ when $\mu=1, x_{\mathrm{R}}=0.4$, and $K_{1}=K_{2}=1.5 \mathrm{~dB}$.

at small $x_{\mathrm{PR}}$, i.e., $\mathrm{PU}$ is near to $\mathrm{S}$, the optimal value $x_{R}^{*}$ is also small. This is due to the fact that $\mathrm{R}$ has to be near to $\mathrm{S}$ to help it relay the signal to D. In Fig. 6, we place PU at different positions, i.e., $x_{\mathrm{PR}}=y_{\mathrm{PR}}=0.05 i, i \in\{1,2, \ldots, 19\}$ and use the Golden Section Search method to find $x_{R}^{*}$ of both protocols in the case that only interference constraint $I_{t h}$ is required.

\section{CONCLUSIONS}

Two important CCN schemes, with and without direct link, have been investigated when the secondary network experiences Rician fading and the SUs $\rightarrow$ PU links are subject to Rayleigh fading. Both exact and asymptotic OP expressions have been obtained for the considered CCNs. The CCN with incremental DF relaying has been shown to outperform both conventional dual-hop DF relaying, which reveals the significance of relay deployment in enhancing the performance of secondary networks, operating under strict power conditions. 


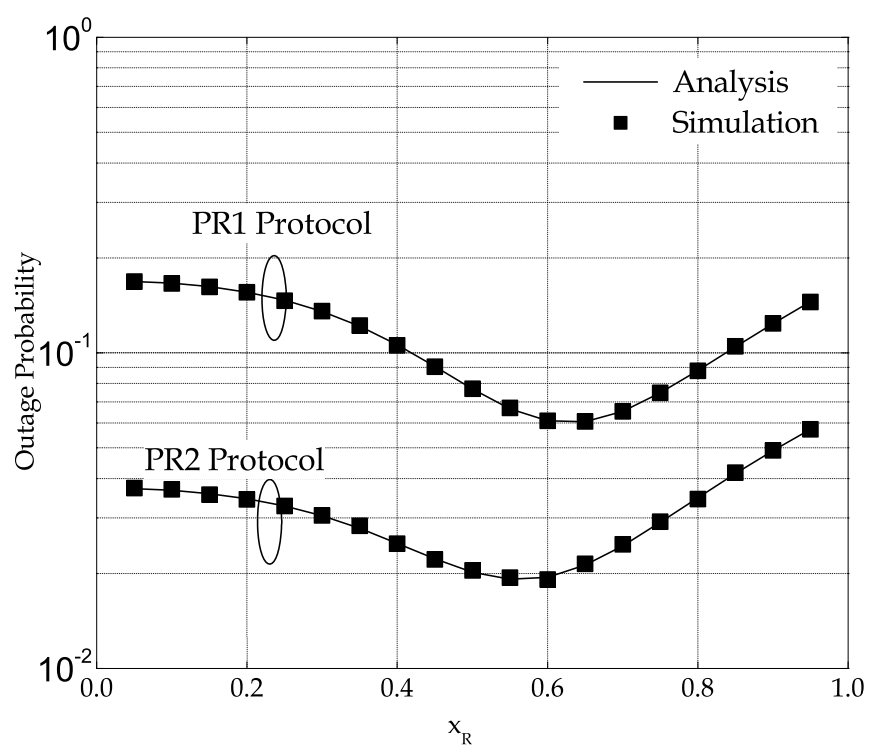

Fig. 4. The OP as a function of $x_{\mathrm{R}}$ without $P_{t h}$ constraint and $x_{\mathrm{PR}}=$ $y_{\mathrm{PR}}=0.5, K_{1}=K_{2}=2 \mathrm{~dB}$, and $\gamma_{P}=10 \mathrm{~dB}$.

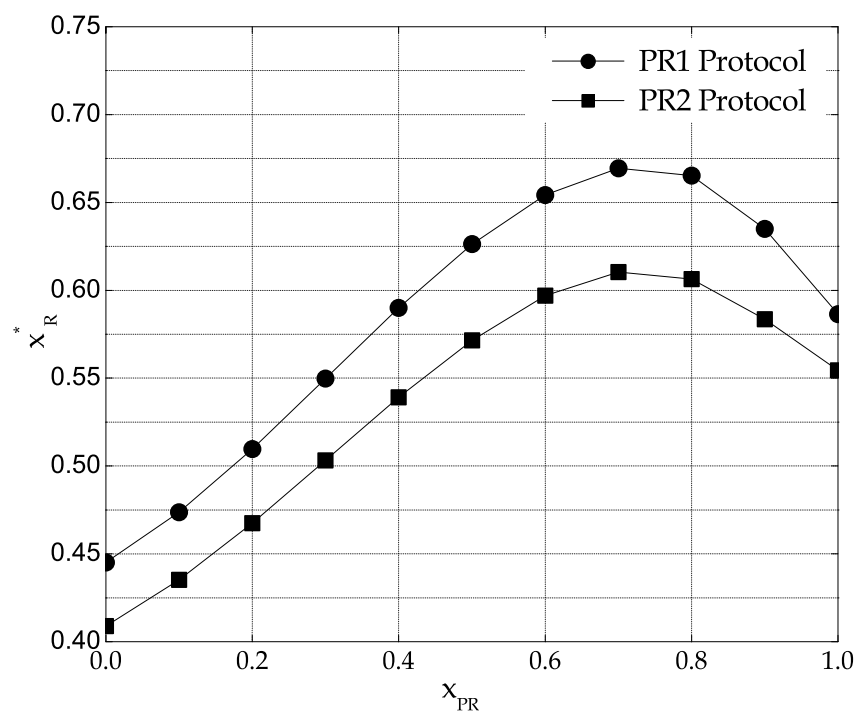

Fig. 5. The optimal value $x_{\mathrm{R}}^{*}$ as a function of $x_{\mathrm{PR}}$ when $y_{\mathrm{PR}}=0.5$, $K_{1}=K_{2}=1 \mathrm{~dB}, \mu=1$, and $\gamma_{P}=10 \mathrm{~dB}$.

\section{REFERENCES}

[1] A. Ghasemi and E. S. Sousa, "Fundamental limits of spectrum-sharing in fading environments," IEEE Trans. Wireless Commun., vol. 6, no. 2, pp. 649-658, Feb. 2007.

[2] A. Giorgetti, M. Varrella, and M. Chiani, "Analysis and performance comparison of different cognitive radio algorithms," in Proc. IEEE Int. Work. on Cognit. Radio Adv. Spectr. Managem. (CogART), Aalborg, Denmark, May 2009, pp. 127-131.

[3] H. A. Suraweera, P. J. Smith, and M. Shafi, "Capacity limits and performance analysis of cognitive radio with imperfect channel knowledge," IEEE Trans. Veh. Technol., vol. 59, no. 4, pp. 1811-1822, Apr. 2010.

[4] B. Maham, P. Popovski, X. Zhou, and A. Hjørungnes, "Cognitive multiple access network with outage margin in the primary system," IEEE Trans. Wireless Commun., vol. 10, no. 10, pp. 3343-3353, Oct. 2011.

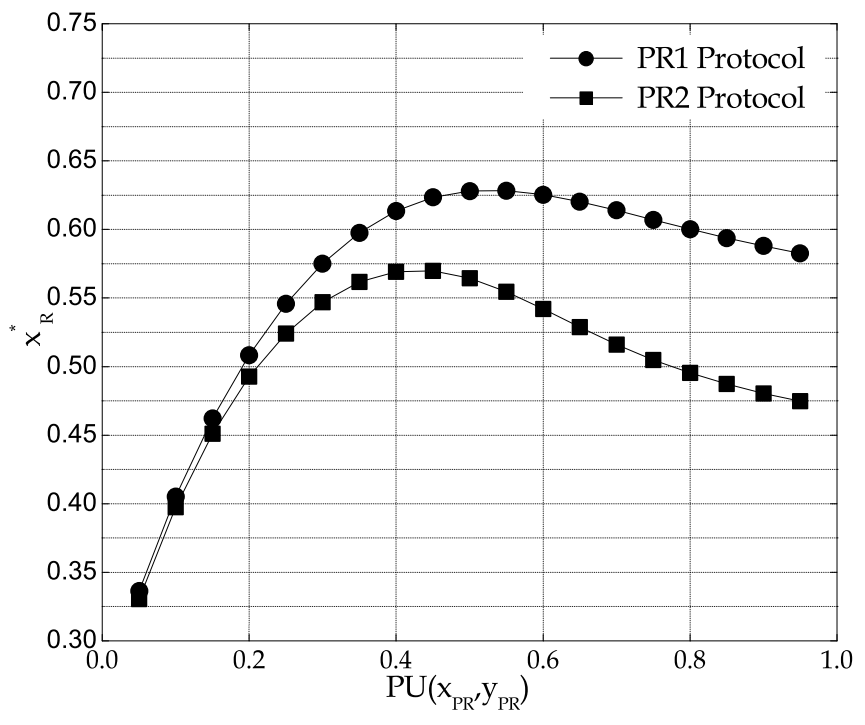

Fig. 6. The optimal value $x_{\mathrm{R}}^{*}$ as a function of PU's positions without $P_{t h}$ constraint and $K_{1}=K_{2}=2 \mathrm{~dB}$, and $\gamma_{P}=10 \mathrm{~dB}$.

[5] H. Ding, J. Ge, D. B. da Costa, and Z. Jiang, "Asymptotic analysis of cooperative diversity systems with relay selection in a spectrum sharing scenario," IEEE Trans. Veh. Technol., vol. 60, no. 2, pp. 457-472, Feb. 2011.

[6] L. B. Le and E. Hossain, "Resource allocation for spectrum underlay in cognitive radio networks," IEEE Trans. Wireless Commun., vol. 7, no. 12 , pp. 5306-5315, Dec. 2008.

[7] J. Lee, H. Wang, J. G. Andrews, and D. Hong, "Outage probability of cognitive relay networks with interference constraints," IEEE Trans. Wireless Commun., vol. 10, no. 2, pp. 390-395, Feb. 2011.

[8] T. Q. Duong, V. N. Q. Bao, G. C. Alexandropoulos, and H.-J. Zepernick, "Cooperative spectrum sharing networks with $\mathrm{AF}$ relay and selection diversity," Electron. Lett., vol. 47, no. 20, pp. 1149-1151, Sep. 2011.

[9] C. Zhong, T. Ratnarajah, and K.-K. Wong, "Outage analysis of decodeand-forward cognitive dual-hop systems with the interference constraint in Nakagami- $m$ fading channels," IEEE Trans. Veh. Technol., vol. 60, no. 7, pp. 2875-2879, July 2011

[10] T. Q. Duong, D. B. da Costa, M. Elkashlan, and V. N. Q. Bao, "Cognitive amplify-and-forward relay networks over Nakagami- $m$ fading," IEEE Trans. Veh. Technol., vol. 61, no. 5, pp. 2368-2374, May 2012.

[11] B. Maham and A. Hjørungnes, "Performance analysis of amplify-andforward opportunistic relaying in Rician fading," IEEE Signal Process. Lett., vol. 16, no. 8, pp. 643-646, Aug. 2009.

[12] P. Kÿosti et al., "WINNER II Interim Channel Models (IST-4-027756 WINNER II D1.1.1 V1.1)." [Online]. Available: http://www.istwinner.org/WINNER2-Deliverables/D1.1.1.pdf.

[13] H. A. Suraweera, G. K. Karagiannidis, and P. J. Smith, "Performance analysis of the dual-hop asymmetric fading channel," IEEE Trans. Wireless Commun., vol. 8, no. 6, pp. 2783-2788, June 2009.

[14] H. A. Suraweera, R. H. Y. Louie, Y. Li, G. K. Karagiannidis, and B. Vucetic, "Two hop amplify-and-forward transmission in mixed Rayleigh and Rician fading channels," IEEE Commun. Lett., vol. 13, no. 4, pp. 227-229, Apr. 2009.

[15] M. K. Simon and M.-S. Alouini, Digital Communication over Fading Channels: A Unified Approach to Performance Analysis. New York: Wiley, 2000.

[16] I. S. Gradshteyn and I. M. Ryzhik, Table of Integrals, Series, and Products, 6th ed. San Diego, CA: Academic, 2000.

[17] J. N. Laneman, D. N. C. Tse, and G. W. Wornell, "Cooperative diversity in wireless networks: Efficient protocols and outage behavior," IEEE Trans. Inf. Theory, vol. 50, no. 12, pp. 3062-3080, Dec. 2004.

[18] E. K. P. Chong and S. H. Zak, An Introduction to Optimization. United States: Wiley, 2008. 